\title{
A 2-order additive fuzzy measure identification method based on intuitionistic fuzzy sets and its application in credit evaluation
}

\author{
Mu Zhang ${ }^{\mathrm{a}, *}$, Si-si Li ${ }^{\mathrm{a}, \mathrm{b}}$ and Bi-bin Zhao ${ }^{\mathrm{a}, \mathrm{b}}$ \\ ${ }^{a}$ School of Big Data Application and Economics, Guizhou University of Finance and Economics, Huaxi University \\ Town, Guiyang, Guizhou, China \\ ${ }^{\mathrm{b}}$ Guizhou Institution for Technology Innovation \& Entrepreneurship Investment, Guizhou University of Finance \\ and Economics, Huaxi University Town, Guiyang, Guizhou, China
}

\begin{abstract}
In view of the problem that it is difficult to quantitatively assess the interactivity between attributes in the identification process of 2-order additive fuzzy measure, this work uses the intuitionistic fuzzy sets (IFSs) to describe and deal with the interactivity between attributes. Firstly, the interactivity between attributes is defined by the supermodular game theory. On this basis, the experts employ the intuitionistic fuzzy number (IFN) to assess the interactivity between attributes, Secondly, the opinions of all experts are aggregated by using the intuitionistic fuzzy weighted average operator (IFWA). Finally, based on the aggregated results, the intuitionistic fuzzy interaction degree between attributes is defined and calculated by the score function of IFN. Thus, a 2-order additive fuzzy measure identification method based on IFSs is further proposed. Based on the proposed method, using the Choquet fuzzy integral as nonlinear integration operator, a multi-attribute decision making (MADM) process is presented. Taking the credit evaluation of the big data listed companies in China as an application example, the feasibility and effectiveness of the proposed method is verified by the analysis results of application example.
\end{abstract}

Keywords: Interactivity between attributes, intuitionistic fuzzy sets, 2-order additive fuzzy measure, choquet fuzzy integral, multi-attribute decision making, credit evaluation

\section{Introduction}

Due to the interaction among attributes, such as complementarity and repeatability, the classical weighted arithmetic mean method is often invalid in the process of multi-attribute decision making (MADM) [1]. In order to overcome this limitation,

\footnotetext{
*Corresponding author. Mu Zhang, School of Big Data Application and Economics, Guizhou University of Finance and Economics, Huaxi University Town, Huaxi District, Guiyang, Guizhou, China. Tel.: +86 1528556 5731; Fax: +86 08518851 0575; E-mail: zhangmu01@163.com.
}

and effectively describe and deal with any interaction among attributes, Sugeno [2] proposed the concept of fuzzy measure and fuzzy integral. Since then, after nearly 20 years of development, the theory of fuzzy measures had been formed [3, 4]. However, in the practical application process, when there are $n$ attributes, the general fuzzy measure generally needs to determine $2^{n}-2$ parameters $[4,5]$. This complexity greatly limits its practical application ability.

In order to reduce the complexity, Sugeno [2] proposed the $\lambda$ fuzzy measure, that only needs to determine $n$ parameters, however, the $\lambda$ fuzzy 
measure can only represent a certain kind of interaction among attributes, which weakens its performance ability. Thereafter, in order to face with the complexity of discrete fuzzy measures, Grabisch [6] proposed the $k$-order additive fuzzy measures, including usual additive measures and fuzzy measures. Every discrete fuzzy measure is a $k$-order additive fuzzy measure for a unique $k$. The $k$-order additive fuzzy measures cover all fuzzy measures with any complexity from classical additive measure $(k=1)$ to general fuzzy measure $(k=n)$. Among them, the 2order additive fuzzy measure not only merely needs to determine $n(n+1) / 2$ parameters, but also merely involves the relative importance of attributes and the interaction between attributes, which well solves the contradiction between complexity and performance ability, so it has been widely used [7].

However, because the interaction between attributes is difficult to explain and understand [8], combined with the uncertainty of decision-makers' cognition, the decision-makers often cannot give accurate quantitative assessment on the interactivity between attributes, but with a certain fuzziness and hesitation, which brings difficulties to decisionmaking. The existing 2 -order additive fuzzy measure identification methods use the subjective methods [9-15] and the objective methods [7, 16, 17] to describe and deal with the interactivity between attributes. The subjective methods are more explanatory than the objective methods, so they have been applied more widely. However, the above methods cannot reflect the fuzziness and hesitation of decision-makers in the assessment of interactivity between attributes.

In [18], Atanassov proposed the concept of intuitionistic fuzzy set (IFS). IFS is an extension of traditional fuzzy set. It considers the information of membership degree, non-membership degree and hesitation degree, so it is more flexible and practical than traditional fuzzy set in dealing with fuzziness and uncertainty [19]. IFS can better and more completely reflect the fuzziness and hesitation of the decision-maker's judgment of the objective things, so it has been successfully applied in the field of uncertain MADM [20-22].

Based on this observation, in view of the problem that it is difficult to quantitatively assess the interactivity between attributes, the present work uses the IFSs to describe and deal with the interactivity between attributes. As a result, a 2-order additive fuzzy measure identification method based on IFSs is proposed. Based on the proposed method, using the Choquet fuzzy integral as nonlinear integration operator [23], a MADM process is further presented.

This paper is structured as follows: Section 2 introduces the preparatory knowledge employed in this work. Section 3 proposes the 2 -order additive fuzzy measure identification method based on IFSs. Section 4 presents the MADM process based on the proposed method. Section 5 describes the analysis results of application example. Section 6 discusses the results obtained and Section 7 concludes this paper.

\section{Preparatory knowledge}

This section introduces the related definitions of IFSs, 2-order additive fuzzy measure and Choquet fuzzy integral reported in the literatures. This is the basis of Section 3 and Section 4.

Definition 1 [18]. Let $X$ be a non-empty set, then $A=$ $\left\{\left\langle x, \mu_{A}(x), v_{A}(x)\right\rangle \mid x \in X\right\}$ is called the IFS, where $\mu_{A}(x)$ and $v_{A}(x)$ are the membership degree and nonmembership degree of element $x$ in $X$ belonging to $A$ respectively, then $\mu_{A}: X \rightarrow[0,1], v_{A}: X \rightarrow[0,1]$ holds, and satisfies $0 \leq \mu_{A}(x)+v_{A}(x) \leq 1, \forall x \in X$. In addition, $\pi_{A}(x)=1-\mu_{A}(x)-v_{A}(x)$ is called the hesitation degree of element $x$ in $X$ belonging to $A$, and satisfies $0 \leq \pi_{A}(x) \leq 1, \forall x \in X$. For convenience, let $\alpha=\left(\mu_{\alpha}, v_{\alpha}\right)$ be an IFN, where $\mu_{\alpha} \in[0,1], v_{\alpha} \in$ $[0,1], 0 \leq \mu_{\alpha}+v_{\alpha} \leq 1$, and let $\Theta$ be the set of all IFNs.

Definition 2 [18]. Let $a_{1}=\left(\mu_{1}, v_{1}\right)$ and $a_{2}=$ $\left(\mu_{2}, \nu_{2}\right)$ be two IFNs on a given domain; let $\lambda$ be a real number, and $\lambda>0$, then the operational rules of IFNs are as follows:

1) $a_{1} \oplus a_{2}=\left(\mu_{1}+\mu_{2}-\mu_{1} \mu_{2}, v_{1} v_{2}\right)$; 2) $a_{1} \otimes$ $\left.a_{2}=\left(\mu_{1} \mu_{2}, v_{1}+v_{2}-v_{1} v_{2}\right) ; 3\right) \lambda a_{1}=(1-(1-$ $\left.\left.\mu_{1}\right)^{\lambda}, v_{1}^{\lambda}\right)$; 4) $a_{1}^{\lambda}=\left(\mu_{1}^{\lambda}, 1-\left(1-v_{1}\right)^{\lambda}\right)$.

Definition 3 [24]. Let $\alpha_{j}=\left(\mu_{\alpha_{j}}, v_{\alpha_{j}}\right)(j=1,2$, $\cdots, n)$ be a set of IFNs, $w_{j}$ is the weight of $\alpha_{j}$, where $w_{j} \in[0,1]$, and $\sum_{j=1}^{n} w_{j}=1$. Let $I F W A$ : $\Theta^{n} \rightarrow \Theta$, if

$$
I F W A_{w}\left(\alpha_{1}, \alpha_{2}, \cdots, \alpha_{n}\right)=\left(1-\prod_{j=1}^{n}\left(1-\mu_{\alpha_{j}}\right)^{w_{j}}, \prod_{j=1}^{n} v_{\alpha_{j}}^{w_{j}}\right)
$$

then IFWA is called the intuitionistic fuzzy weighted average operator. 
Definition 4 [25]. Let $U=\left\{x_{1}, x_{2}, \cdots, x_{n}\right\}$ be a given domain, $A=\sum_{i=1}^{n}\left\langle\mu_{A}\left(x_{i}\right), v_{A}\left(x_{i}\right)\right\rangle / x_{i}$ is an IFS, then the intuitionistic fuzzy entropy of $A$ is

$$
E(A)=\frac{1}{n} \sum_{i=1}^{n} \frac{\min \left(\mu_{A}\left(x_{i}\right), v_{A}\left(x_{i}\right)\right)+\pi_{A}\left(x_{i}\right)}{\max \left(\mu_{A}\left(x_{i}\right), v_{A}\left(x_{i}\right)\right)+\pi_{A}\left(x_{i}\right)}
$$

Definition 5 [26, 27]. Let $\alpha=\left(\mu_{\alpha}, v_{\alpha}\right)$ be an IFN, where $\mu_{\alpha} \in[0,1], v_{\alpha} \in[0,1], 0 \leq \mu_{\alpha}+v_{\alpha} \leq 1$, when the hesitation degree is not considered, the score function of IFN $\alpha$ can be expressed as [26]

$$
S(\alpha)=\mu_{\alpha}-v_{\alpha}, \quad S(\alpha) \in[-1,1]
$$

When considering the hesitation degree, the score function of IFN $\alpha$ can be expressed as [27]

$$
S_{L}(\alpha)=\mu_{\alpha}+\mu_{\alpha}\left(1-\mu_{\alpha}-v_{\alpha}\right), \quad S_{L}(\alpha) \in[0,1]
$$

Definition 6 [2, 6]. Let $X=\left\{x_{1}, x_{2}, \cdots, x_{n}\right\}$ be a set of attributes, let $X^{*}=\{1,2, \cdots, n\}$ be a set of subscripts of attributes. $P(X)$ is the power set of $X$. If the set function $g: P(X) \rightarrow[0,1]$ satisfies the following two conditions:

1) $g(\varnothing)=0, g(X)=1$; 2) If $K \in P(X), T \in$ $P(X), K \subseteq T$, then $g(K) \leq g(T)$.

then $g$ is called a fuzzy measure on $P(X)$ [2].

Grabisch [6] proposed the $k$-order additive fuzzy measures based on pseudo-Boolean function and Möbius transformation. On this basis, the 2-order additive fuzzy measure is then defined as

$$
g(K)=\sum_{i \in K^{*}} m_{i}+\sum_{\{i, j\} \subset K^{*}} m_{i j}, \forall K \subseteq X
$$

where $m_{i}$ is the Möbius transformation coefficient of $x_{i}(i=1,2, \cdots, n)$, which is an overall importance; $m_{i j}$ is the Möbius transformation coefficient of $\left\{x_{i}, x_{j}\right\}(i, j=1,2, \cdots, n ; i \neq j)$, which represents the extent of interaction between $x_{i}$ and $x_{j}$.

Definition 7 [13]. Let $X=\left\{x_{1}, x_{2}, \cdots, x_{n}\right\}$ be a set of attributes, $W=\left\{w_{1}, w_{2}, \cdots, w_{n}\right\}$ is the weight set of $X$, the Möbius transformation coefficients of $x_{i}$ and $\left\{x_{i}, x_{j}\right\}$ are respectively

$$
\left\{\begin{array}{c}
m_{i}=w_{i} / P \\
m_{i j}=\xi_{i j} w_{i} w_{j} / P
\end{array}, \quad i, j=1,2, \cdots, n ; i \neq j\right.
$$

where $P=\sum_{i \in X^{*}} w_{i}+\sum_{\{i, j\} \subset X^{*}} \xi_{i j} w_{i} w_{j}$ is the sum of the importance of all $x_{i}$ and $\left\{x_{i}, x_{j}\right\}, \xi_{i j}$ is the interaction degree between $x_{i}$ and $x_{j}, \xi_{i j}$ $\in[-1,1]$.

Definition 8 [23]. Let $f$ be a nonnegative function defined on $X, F$ is a $\sigma-$ algebra composed of subsets of $X$ (when $X$ is finite, $F$ is the power set $P(X)$ of $X$ ), $g$ is a fuzzy measure defined on $F$, then the Choquet fuzzy integral of function $f$ on set $X$ for fuzzy measure $g$ is defined as $(c) \int f d g=\int_{0}^{\infty} g\left(F_{\alpha}\right) d \alpha$, where $F_{\alpha}=$ $\{x \mid f(x) \geq \alpha, x \in X\}, \alpha \in[0, \infty] ; \int_{0}^{\infty} g\left(F_{\alpha}\right) d \alpha$ is the Riemann integral.

When $X$ is a finite set, the elements in $X$ are rearranged as $\left\{x_{(1)}, x_{(2)}, \cdots, x_{(n)}\right\}$, which makes $f\left(x_{(1)}\right) \leq f\left(x_{(2)}\right) \leq \cdots \leq f\left(x_{(n)}\right)$. Let $H=$ (c) $\int f d g$, then the Choquet fuzzy integral has the following simplified formula:

$$
H=(c) \int f d g=\sum_{i=1}^{n}\left[f\left(x_{(i)}\right)-f\left(x_{(i-1)}\right)\right] g\left(X_{(i)}\right)
$$

where $X_{(i)}=\left\{x_{(i)}, x_{(i+1)}, \cdots, x_{(n)}\right\},(i)=(1),(2)$, $\cdots,(n) ; f\left(x_{(0)}\right)=0$.

\section{A 2-order additive fuzzy measure identification method based on IFSs}

Based on IFSs, this section proposes a 2-order additive fuzzy measure identification method. In addition, the correctness of the proposed method is proved theoretically.

Let $A=\left\{A_{1}, A_{2}, \cdots, A_{m}\right\}$ be a finite set of alternatives, and $C=\left\{C_{1}, C_{2}, \cdots, C_{n}\right\}$ be a set of attributes to compare the alternatives, let $C^{*}=\{1,2, \cdots, n\}$ be a set of subscripts of attributes. The weight vector of attributes is $W_{C}=\left(w_{1}, w_{2}, \cdots, w_{n}\right)$, where $w_{i} \in[0,1]$, and $\sum_{i=1}^{n} w_{i}=1$. Let $D=\left\{D_{1}, D_{2}, \cdots, D_{p}\right\}$ be a set of experts, the weight vector of experts is $W_{D}=\left(w_{1}, w_{2}, \cdots, w_{p}\right)$, where $w_{l} \in[0,1]$, and $\sum_{l=1}^{p} w_{l}=1$. Based on IFSs, the identification process of 2-order additive fuzzy measure is as follows:

Step 1: Defines the interactivity between attributes.

According to the supermodular game theory, the interactivity between attributes is defined as follows:

Definition 9. Let any two attributes $C_{i}$ and $C_{j}(i \neq j)$ in attribute set $C$ have partial order relation, the supremum $C_{i} \vee C_{j}$ and the infimum $C_{i} \wedge C_{j}$ are in $C$, then $C$ is called a sub-lattice [28]. Let $f$ be a realvalued function defined on the sub-lattice $C, C \subseteq R^{n}$. 
For $\forall C_{i}, C_{j} \in C$, when $f\left(C_{i} \vee C_{j}\right)+f\left(C_{i} \wedge C_{j}\right)>$ $f\left(C_{i}\right)+f\left(C_{j}\right), f$ is a supermodular function [28], then it is said that there is complementarity between $C_{i}$ and $C_{j}(i \neq j)$; when $f\left(C_{i} \vee C_{j}\right)+f\left(C_{i} \wedge C_{j}\right)<$ $f\left(C_{i}\right)+f\left(C_{j}\right), f$ is a submodular function [28], then it is said that there is repeatability between $C_{i}$ and $C_{j}(i \neq j)$; particularly, when $f\left(C_{i} \vee C_{j}\right)+f\left(C_{i} \wedge\right.$ $\left.C_{j}\right)=f\left(C_{i}\right)+f\left(C_{j}\right)$, then it is said that there is independence between $C_{i}$ and $C_{j}(i \neq j)$.

Step 2: Calculate the individual assessment result of each expert on the interactivity between $C_{i}$ and $C_{j}$ $(i \neq j)$.

According to Definition 9, the expert $D_{l}(l=$ $1,2, \cdots, p)$ employs the IFN $\alpha=\left(\mu_{\alpha}, v_{\alpha}\right)$ to assess the interactivity between $C_{i}$ and $C_{j}(i \neq j)\left(C_{n}^{2}\right.$ pairs in total). When the expert thinks that there is complementarity between $C_{i}$ and $C_{j}(i \neq j), \mu_{\alpha}>0.5$, and the larger $\mu_{\alpha}$ is, the stronger complementarity is. When the expert thinks that there is repeatability between $C_{i}$ and $C_{j}(i \neq j), v_{\alpha}>0.5$, and the larger $v_{\alpha}$ is, the stronger repeatability is. In addition, when the expert thinks that $C_{i}$ and $C_{j}(i \neq j)$ are independent of each other, $\mu_{\alpha}=v_{\alpha}=0.5$. Thus, the individual assessment result $\tilde{\xi}_{i j l}=\left(\mu_{\tilde{\xi}_{i j l}}, v_{\tilde{\xi}_{i j l}}\right)(l=$ $1,2, \cdots, p)$ of the interactivity between $C_{i}$ and $C_{j}$ $(i \neq j)$ is then given.

Step 3: Calculate the group assessment result of $p$ experts on the interactivity between $C_{i}$ and $C_{j}$ $(i \neq j)$.

Given the weight vector $W_{D}$ of experts, using Equation (1) to aggregate the opinions of $p$ experts, the group assessment result $\tilde{\xi}_{i j}$ of the interactivity between $C_{i}$ and $C_{j}(i \neq j)$ is further calculated as

$$
\tilde{\xi}_{i j}=\left(\mu_{\tilde{\xi}_{i j}}, \nu_{\tilde{\xi}_{i j}}\right)=\left(1-\prod_{l=1}^{p}\left(1-\mu_{\tilde{\xi}_{i j l}}\right)^{w_{l}}, \prod_{l=1}^{p} v_{\tilde{\xi}_{i j l}}^{w_{l}}\right)
$$

Step 4: Determine the intuitionistic fuzzy interaction degree $S\left(\tilde{\xi}_{i j}\right)$ between $C_{i}$ and $C_{j}(i \neq j)$.

With Equation (3), the intuitionistic fuzzy interaction degree $S\left(\tilde{\xi}_{i j}\right)$ between $C_{i}$ and $C_{j}(i \neq j)$ is defined as follows:

Definition 10. Let $\tilde{\xi}_{i j}=\left(\mu_{\tilde{\xi}_{i j}}, v_{\tilde{\xi}_{i j}}\right)$ be the group assessment result of the interactivity between $C_{i}$ and $C_{j}(i \neq j)$, according to Equation (3), if $S\left(\tilde{\xi}_{i j}\right)$ satisfies

$$
S\left(\tilde{\xi}_{i j}\right)=\mu_{\tilde{\xi}_{i j}}-v_{\tilde{\xi}_{i j}}, \quad S\left(\tilde{\xi}_{i j}\right) \in[-1,1]
$$

then $S\left(\tilde{\xi}_{i j}\right)$ is called the intuitionistic fuzzy interaction degree between $C_{i}$ and $C_{j}(i \neq j)$. If there is comple- mentarity between $C_{i}$ and $C_{j}(i \neq j)$, then $S\left(\tilde{\xi}_{i j}\right)>0$, and the larger $S\left(\tilde{\xi}_{i j}\right)$ is, the stronger complementarity is. If there is repeatability between $C_{i}$ and $C_{j}(i \neq j)$, then $S\left(\tilde{\xi}_{i j}\right)<0$, and the smaller $S\left(\tilde{\xi}_{i j}\right)$ is, the stronger repeatability is. If $C_{i}$ and $C_{j}(i \neq j)$ are independent of each other, then $S\left(\tilde{\xi}_{i j}\right)=0$.

Thus, according to Definition 10, the intuitionistic fuzzy interaction degree $S\left(\tilde{\xi}_{i j}\right)$ between $C_{i}$ and $C_{j}$ $(i \neq j)$ can be determined.

Step 5: Calculate the Möbius transformation coefficients $m_{i}$ and $m_{i j}$ of attributes.

According to the weight $w_{i}$ of the $i$-th attribute and the intuitionistic fuzzy interaction degree $S\left(\tilde{\xi}_{i j}\right)$ between $C_{i}$ and $C_{j}(i \neq j)$, using Equation (6), the Möbius transformation coefficients $m_{i}$ and $m_{i j}$ of attributes can be calculated as

$$
\left\{\begin{array}{c}
m_{i}=w_{i} / P \\
m_{i j}=S\left(\tilde{\xi}_{i j}\right) w_{i} w_{j / P}, \quad i, j=1,2, \cdots, n ; i \neq j
\end{array}\right.
$$

where $P=\sum_{i \in C^{*}} w_{i}+\sum_{\{i, j\} \subset C^{*}} S\left(\tilde{\xi}_{i j}\right) w_{i} w_{j}$ is the sum of the importance of all $C_{i}$ and $\left\{C_{i}, C_{j}\right\}(i \neq j)$.

Step 6: Identify the 2-order additive fuzzy measure $g_{K}$.

According to the Möbius transformation coefficients $m_{i}$ and $m_{i j}$ of attributes, using Equation (5), the 2-order additive fuzzy measure $g_{K}$ can be identified as

$$
g_{K}=g(K)=\sum_{i \in K^{*}} m_{i}+\sum_{\{i, j\} \subset K^{*}} m_{i j}, \forall K \subseteq C
$$

Theorem 1. The fuzzy measure identified by steps 1 to 6 is a 2-order additive fuzzy measure.

To prove that the fuzzy measure identified by steps 1 to 6 is a 2-order additive fuzzy measure, it is only necessary to prove that the determined Möbius transformation coefficients satisfy the following constrained conditions [6]:

$$
\begin{aligned}
\text { 1) } m(\varnothing) & =0 \text {;2) } m_{i} \geq 0, \forall i \in C^{*} \text {;3) } \sum_{i \in C^{*}} m_{i}+ \\
\sum_{\{i, j\} \subset C^{*}} m_{i j} & =1 \text {; 4) } m_{i}+\sum_{j \in K^{*} \backslash\{i\}} m_{i j} \geq 0, \forall K \subset C .
\end{aligned}
$$

\section{Proof.}

1) $m(\varnothing)=0$, obviously holds.

2) Because $S\left(\tilde{\xi}_{i j}\right)=S\left(\tilde{\xi}_{j i}\right)$, and $\sum_{i=1}^{n} w_{i}=1, P$ can be further written as 


$$
\begin{aligned}
P & =1+\frac{1}{2} \sum_{i=1}^{n} \sum_{j=1, i \neq j}^{n} S\left(\tilde{\xi}_{i j}\right) w_{i} w_{j} \\
& =1+\frac{1}{2} \sum_{i=1}^{n}\left[w_{i} \sum_{j=1, i \neq j}^{n} S\left(\tilde{\xi}_{i j}\right) w_{j}\right]
\end{aligned}
$$

Since $S\left(\tilde{\xi}_{i j}\right) \in[-1,1]$, and $w_{j} \in[0,1]$, we have

$$
-w_{j} \leq S\left(\tilde{\xi}_{i j}\right) w_{j} \leq w_{j}, i \neq j
$$

Sum the two sides of the above inequality to $j$, we obtain

$$
-\left(1-w_{i}\right) \leq \sum_{j=1, i \neq j}^{n} S\left(\tilde{\xi}_{i j}\right) w_{j} \leq 1-w_{i}
$$

Multiply both sides of the above inequality by $w_{i}$, we can get

$$
-\left(w_{i}-w_{i}^{2}\right) \leq w_{i} \sum_{j=1, i \neq j}^{n} S\left(\tilde{\xi}_{i j}\right) w_{j} \leq w_{i}-w_{i}^{2}
$$

Sum the two sides of the above inequality to $i$, the following inequality can be given

$$
\begin{aligned}
-\left(1-\sum_{i=1}^{n} w_{i}^{2}\right) & \leq \sum_{i=1}^{n}\left[w_{i} \sum_{j=1, i \neq j}^{n} S\left(\tilde{\xi}_{i j}\right) w_{j}\right] \\
& \leq 1-\sum_{i=1}^{n} w_{i}^{2}
\end{aligned}
$$

Therefore, we have

$$
\begin{aligned}
\frac{1}{2}+\frac{1}{2} \sum_{i=1}^{n} w_{i}^{2} & \leq 1+\frac{1}{2} \sum_{i=1}^{n}\left[w_{i} \sum_{j=1, i \neq j}^{n} S\left(\tilde{\xi}_{i j}\right) w_{j}\right] \\
& \leq \frac{3}{2}-\frac{1}{2} \sum_{i=1}^{n} w_{i}^{2}
\end{aligned}
$$

That is to say

$$
0<\frac{1}{2}+\frac{1}{2} \sum_{i=1}^{n} w_{i}^{2} \leq P
$$
0 .

Because $P>0$, and $w_{i} \geq 0$, we can get $m_{i}=\frac{w_{i}}{P} \geq$

3) $\sum_{i \in C^{*}} m_{i}+\sum_{\{i, j\} \subset C^{*}} m_{i j}=$

$\sum_{i \in C^{*}} \frac{w_{i}}{P}+\sum_{\{i, j\} \subset C^{*}} \frac{S\left(\tilde{\xi}_{i j}\right) w_{i} w_{j}}{P}=$

$\frac{1}{P}\left[\sum_{i \in C^{*}} w_{i}+\sum_{\{i, j\} \subset C^{*}} S\left(\tilde{\xi}_{i j}\right) w_{i} w_{j}\right]=1$, obviously holds.

$$
\text { 4) } \begin{aligned}
& m_{i}+\sum_{j \in K^{*} \backslash\{i\}} m_{i j}=\frac{w_{i}}{P}+\sum_{j \in K^{*} \backslash\{i\}} \frac{S\left(\tilde{\xi}_{i j}\right) w_{i} w_{j}}{P}= \\
& \frac{w_{i}}{P}\left[1+\sum_{j \in K^{*} \backslash\{i\}} S\left(\tilde{\xi}_{i j}\right) w_{j}\right]
\end{aligned}
$$

Since $S\left(\tilde{\xi}_{i j}\right) \in[-1,1]$, and $w_{j} \in[0,1]$, we have $-\left(1-w_{i}\right) \leq \sum_{j \in K^{*} \backslash\{i\}} S\left(\tilde{\xi}_{i j}\right) w_{j} \leq 1-w_{i}$. Thus, the following inequality can be given

$$
\begin{aligned}
& \frac{w_{i}}{P}\left[1+\sum_{j \in K^{*} \backslash\{i\}} S\left(\tilde{\xi}_{i j}\right) w_{j}\right] \\
& \geq \frac{w_{i}}{P}\left[1-\left(1-w_{i}\right)\right] \geq \frac{w_{i}^{2}}{P} \geq 0
\end{aligned}
$$

Hence, we get $m_{i}+\sum_{j \in K^{*} \backslash\{i\}} m_{i j} \geq 0, \forall K \subset C$. Q.E.D.

\section{A MADM process based on the proposed method}

Using the Choquet fuzzy integral as nonlinear integration operator, this section presents a MADM process based on the proposed method.

Step 1: Calculate the group intuitionistic fuzzy assessment value $\tilde{x}_{j i k}$.

Let $C_{i}=\left\{C_{i 1}, C_{i 2}, \cdots, C_{i k}, \cdots, C_{i n_{i}}\right\} \quad(k=1$, $\left.2, \cdots, n_{i}\right)$ be a set of sub-attributes under the $i$-th attribute. According to the type of sub-attribute (such as positive, negative and neutral), the expert $D_{l}(l=$ $1,2, \cdots, p)$ employs the IFN to assess the performance of the alternative $A_{j}(j=1,2, \cdots, m)$ under the sub-attribute $C_{i k}(i=1,2, \cdots, n)$, the individual intuitionistic fuzzy assessment value $\tilde{x}_{j i k l}=$ $\left(\mu_{\tilde{x}_{j i k l}}, v_{\tilde{x}_{j i k l}}\right)$ of the $k$-th sub-attribute of the alternative $A_{j}$ under the $i$-th attribute is then given.

Given the weight vector $W_{D}$ of experts, using Equation (1) to aggregate the opinions of $p$ experts, the group intuitionistic fuzzy assessment value $\tilde{x}_{j i k}$ of the $k$-th sub-attribute of the alternative $A_{j}$ under the $i$-th attribute is further calculated as

$$
\tilde{x}_{j i k}=\left(\mu_{\tilde{x}_{j i k}}, v_{\tilde{x}_{j i k}}\right)=\left(1-\prod_{l=1}^{p}\left(1-\mu_{\tilde{x}_{j i k l}}\right)^{w_{l}}, \prod_{l=1}^{p} v_{\tilde{x}_{j i k l}}^{w_{l}}\right)
$$


Step 2: Calculate the overall weight $w_{i}$ of the $i$ th attribute and the relative weight $w_{i k}^{\prime}$ of the $k$-th sub-attribute under the $i$-th attribute.

By using Equation (2), the intuitionistic fuzzy entropy $E_{i k}$ of the $k$-th sub-attribute under the $i$-th attribute is calculated as

$$
E_{i k}=\frac{1}{m} \sum_{j=1}^{m} \frac{\min \left(\mu_{\tilde{x}_{j i k}}, v_{\tilde{x}_{j i k}}\right)+\pi_{\tilde{x}_{j i k}}}{\max \left(\mu_{\tilde{x}_{j i k}}, v_{\tilde{x}_{j i k}}\right)+\pi_{\tilde{x}_{j i k}}}
$$

According to the definition of information entropy, if the information entropy of an attribute is smaller, the information quantity provided by the attribute is larger, and the weight of the attribute is also greater [29]. Therefore, the entropy weight $w_{i k}$ of the $k$-th sub-attribute under the $i$-th attribute can be expressed as [30]

$$
w_{i k}=\frac{1-E_{i k}}{\sum_{i=1}^{n} n_{i}-\sum_{i=1}^{n} \sum_{k=1}^{n_{i}} E_{i k}}
$$

That is to say, the overall weight vector of subattributes is $W_{i}=\left(w_{i 1}, w_{i 2}, \cdots, w_{i k}, \cdots, w_{i n_{i}}\right), i=$ $1,2, \cdots, n$. The overall weight $w_{i}$ of the $i$-th attribute is then obtained as

$$
w_{i}=\sum_{k=1}^{n_{i}} w_{i k}
$$

It can be proved that $\sum_{i=1}^{n} w_{i}=1$ holds. Therefore, the overall weight vector of attributes is $W_{C}=$ $\left(w_{1}, w_{2}, \cdots, w_{i}, \cdots, w_{n}\right)$. The relative weight $w_{i k}^{\prime}$ of the $k$-th sub-attribute under the $i$-th attribute is then obtained as

$$
w_{i k}^{\prime}=\frac{w_{i k}}{w_{i}}
$$

Step 3: Calculate the intuitionistic fuzzy assessment score $S_{L}\left(\tilde{x}_{j i}\right)$ of the alternative $A_{j}$ under the $i$-th attribute.

According to $\tilde{x}_{j i k}=\left(\mu_{\tilde{x}_{j i k}}, v_{\tilde{x}_{j i k}}\right)$ and $w_{i k}^{\prime}$, with Equation (1), the intuitionistic fuzzy assessment value $\tilde{x}_{j i}$ of the alternative $A_{j}$ under the $i$-th attribute is calculated as

$\tilde{x}_{j i}=\left(\mu_{\tilde{x}_{j i}}, v_{\tilde{x}_{j i}}\right)=\left(1-\prod_{k=1}^{n_{i}}\left(1-\mu_{\tilde{x}_{j i k}}\right)^{w_{i k}^{\prime}}, \prod_{k=1}^{n_{i}} v_{\tilde{x}_{j i k}}^{w_{i k}^{\prime}}\right)$

Hence, with Equation (4), the intuitionistic fuzzy assessment score $S_{L}\left(\tilde{x}_{j i}\right)$ of the alternative $A_{j}$ under the $i$-th attribute is further calculated as

$$
S_{L}\left(\tilde{x}_{j i}\right)=\mu_{\tilde{x}_{j i}}+\mu_{\tilde{x}_{j i}}\left(1-\mu_{\tilde{x}_{j i}}-v_{\tilde{x}_{j i}}\right), S_{L}\left(\tilde{x}_{j i}\right) \in[0,1]
$$

Step 4: Identify the 2-order additive fuzzy measure $g_{K}$.

Given the weight vector $W_{D}$ of experts, the intuitionistic fuzzy interaction degree $S\left(\tilde{\xi}_{i j}\right)$ between $C_{i}$ and $C_{j}(i \neq j)$ can be determined by steps 1 to 4 in Section 3. Thus, according to $w_{i}$ and $S\left(\tilde{\xi}_{i j}\right)$, the 2-order additive fuzzy measure $g_{K}$ can be further identified by step 5 and step 6 in Section 3 .

Step 5: Calculate the Choquet fuzzy integral values and the ranking of alternatives.

Reordering the intuitionistic fuzzy assessment score $S_{L}\left(\tilde{x}_{j i}\right)(i=1,2, \cdots, n)$ of the alternative $A_{j}$ $(j=1,2, \cdots, m)$ under the $i$-th attribute from small to large, the $S_{L}\left(\tilde{x}_{j(i)}\right)$ can be then obtained. Substituting the $S_{L}\left(\tilde{x}_{j(i)}\right)$ and the 2-order additive fuzzy measure $g_{K}$ into Equation (7), the Choquet fuzzy integral value $H_{j}$ of the alternative $A_{j}$ can be calculated. Simultaneously, the ranking of alternatives can be given, where the larger $H_{j}$ is, the better the alternative $A_{j}$ is.

\section{Application example}

This section takes the credit evaluation of the big data listed companies in China as an application example to illustrate the feasibility and effectiveness of the proposed method (see Section 3).

\subsection{Credit evaluation Index system and sample data}

Considering the characteristics of big data enterprises [31], and following the principles of selecting indicators, such as scientificalness, objectivity, systematization, functionality, dynamics, relative independence, feasibility (or operability), comparability and so on, a credit evaluation index system for big data enterprises was constructed, as shown in Table 1.

We selected the big data listed companies in the Growth Enterprise Market (GEM) in China - Wangsu Science \& Technology Co., Ltd. (300017), Beijing Lanxum Technology Co., Ltd. (300010) and Wuhan Tianyu Information Industry Co., Ltd. (300205) to form a set of alternatives, denoted by $A=A_{1}, A_{2}, A_{3}$. Where the alternative $A_{1}$ and $A_{2}$ belong to the software service industry, and the alternative $A_{3}$ belongs to the electronic components industry. The sample data were the section data of 2016, and the original data were shown in Table 2. Where the original 
Table 1

Credit evaluation index system for big data enterprises

\begin{tabular}{ll}
\hline $\begin{array}{l}\text { Primary } \\
\text { Indicators }\end{array}$ & Secondary Indicators \\
\hline $\begin{array}{l}\text { Debt Paying } \\
\text { Ability } C_{1}\end{array}$ & $\begin{array}{l}\text { Current Ratio } C_{11} \text {; Quick Ratio } C_{12} ; \\
\text { Operational }\end{array}$ \\
Capability $C_{2}$ & $\begin{array}{l}\text { Turnover Rate of Accounts Receivable } C_{21} ; \\
\text { Turnover Rate of Total Assets } C_{22} ; \text { Inventory } \\
\text { Turnover } C_{23}\end{array}$ \\
Profitability & Profit Margin of Main Business $C_{31}$; Return \\
$C_{3}$ & on Equity $C_{32} ;$ Return on Total Assets $C_{33}$ \\
Growth & Net Profit Growth Rate $C_{41}$; Growth Rate of \\
Capability $C_{4}$ & Main Business $C_{42}$ \\
Technological & Development Expenditure $C_{51} ;$ Growth Rate \\
Innovation & of Intangible Assets $C_{52} ;$ Number of \\
Capability $C_{5}$ & Invention Patent Applications Announced \\
Industry & $C_{53}$ \\
Growth $C_{6}$ & Network Attention of Industry $C_{61}$; Industry \\
\hline
\end{tabular}

Table 2

Original data

\begin{tabular}{lccc}
\hline $\begin{array}{l}\text { Secondary Indicators } \\
\text { (Unit, Types) }\end{array}$ & $A_{1}$ & $A_{2}$ & $A_{3}$ \\
\hline$C_{11}\left(-{ }^{*}\right)$ & 6.73 & 1.67 & 2.48 \\
$C_{12}\left(-{ }^{*}\right)$ & 6.49 & 1.38 & 1.76 \\
$C_{13}(\%,-)$ & 15.08 & 28.52 & 33.54 \\
$C_{21}($ time, +$)$ & 5.48 & 2.81 & 3.40 \\
$C_{22}($ time, +$)$ & 0.73 & 0.37 & 0.97 \\
$C_{23}$ (time, +$)$ & 17.32 & 2.78 & 2.88 \\
$C_{31}(\%,+)$ & 28.07 & 15.94 & 0.28 \\
$C_{32}(\%,+)$ & 17.08 & 5.21 & 1.79 \\
$C_{33}(\%,+)$ & 20.52 & 5.83 & 0.27 \\
$C_{41}(\%,+)$ & 50.52 & 130.25 & 12.38 \\
$C_{42}(\%,+)$ & 51.67 & 84.10 & 9.71 \\
$C_{51}(10$ thousand & 5605 & 9868 & 0 \\
yuan, + ( ) & & & \\
$C_{52}(\%,+)$ & 60.57 & 51.24 & -6.73 \\
$C_{53}($ piece, +$)$ & 83 & 0 & 24 \\
$C_{61}($ time, +$)$ & 159 & 159 & 457 \\
$C_{62}(\%,+)$ & 30.77 & 30.77 & 33.42 \\
\hline
\end{tabular}

Note: (1) “+” indicates positive index, “_” indicates negative index, “*” indicates neutral index; (2)The Network Attention of Industry is the overall daily average of Baidu Search Index with the industry name as the key word.

data of the Number of Invention Patent Applications Announced were from Tian Yan Cha website, the original data of the Network Attention of Industry were from Baidu Index website, and the rest of the original data were from East Money website.

\subsection{Process and results of credit evaluation}

Step 1: Calculate the group intuitionistic fuzzy assessment value $\tilde{x}_{j i k}$ of the $k$-th sub-attribute of the alternative $A_{j}$ under the $i$-th attribute.
Table 3

Group intuitionistic fuzzy assessment values of sub-attributes

\begin{tabular}{lccc}
\hline $\begin{array}{l}\text { Sub- } \\
\text { attributes }\end{array}$ & $A_{1}$ & $A_{2}$ & $A_{3}$ \\
\hline$C_{11}$ & $(0.399,0.429)$ & $(0.868,0.108)$ & $(0.800,0.150)$ \\
$C_{12}$ & $(0.248,0.664)$ & $(0.868,0.108)$ & $(0.704,0.183)$ \\
$C_{13}$ & $(0.551,0.266)$ & $(0.720,0.178)$ & $(0.763,0.164)$ \\
$C_{21}$ & $(0.868,0.108)$ & $(0.610,0.226)$ & $(0.704,0.183)$ \\
$C_{22}$ & $(0.704,0.183)$ & $(0.308,0.565)$ & $(0.763,0.164)$ \\
$C_{23}$ & $(0.950,0.050)$ & $(0.566,0.255)$ & $(0.566,0.255)$ \\
$C_{31}$ & $(0.704,0.183)$ & $(0.500,0.300)$ & $(0.050,0.950)$ \\
$C_{32}$ & $(0.924,0.070)$ & $(0.459,0.350)$ & $(0.248,0.664)$ \\
$C_{33}$ & $(0.950,0.050)$ & $(0.566,0.255)$ & $(0.050,0.950)$ \\
$C_{41}$ & $(0.763,0.164)$ & $(0.950,0.050)$ & $(0.551,0.266)$ \\
$C_{42}$ & $(0.763,0.164)$ & $(0.950,0.050)$ & $(0.459,0.350)$ \\
$C_{51}$ & $(0.596,0.235)$ & $(0.868,0.108)$ & $(0.050,0.950)$ \\
$C_{52}$ & $(0.924,0.070)$ & $(0.868,0.108)$ & $(0.050,0.950)$ \\
$C_{53}$ & $(0.924,0.070)$ & $(0.050,0.950)$ & $(0.566,0.255)$ \\
$C_{61}$ & $(0.610,0.226)$ & $(0.610,0.226)$ & $(0.924,0.070)$ \\
$C_{62}$ & $(0.763,0.164)$ & $(0.763,0.164)$ & $(0.885,0.097)$ \\
\hline
\end{tabular}

According to the type of sub-attribute (such as positive, negative and neutral), three experts employed the IFN to assess the performance of the alternative $A_{j}$ under the sub-attribute $C_{i k}$ respectively, the individual intuitionistic fuzzy assessment value $\tilde{x}_{j i k l}$ of the $k$-th sub-attribute of the alternative $A_{j}$ under the $i$-th attribute was then given.

Adopting the cycle mutual evaluation method [32], the weight vector of experts was calculated as $W_{D}=$ $(0.3976,0.3012,0.3012)$ (see Appendix A for full calculation principle and process).

Using Equation (12) to aggregate the opinions of three experts, the group intuitionistic fuzzy assessment value $\tilde{x}_{j i k}$ of the $k$-th sub-attribute of the alternative $A_{j}$ under the $i$-th attribute was further obtained, as shown in Table 3.

Step 2: Determine the weights of attributes.

Based on Table 3, with Equations (13) and (14), the intuitionistic fuzzy entropy $E_{i k}$ and entropy weight $w_{i k}$ of the $k$-th sub-attribute under the $i$-th attribute were calculated. In addition, with Equation (15), the overall weight vector $W_{C}$ of attributes was also obtained. With Equation (16), the relative weight $w_{i k}^{\prime}$ of the $k$-th sub-attribute under the $i$-th attribute was then obtained. The above calculation results were shown in Table 4.

Step 3: Calculate the intuitionistic fuzzy assessment value $\tilde{x}_{j i}$ of the alternative $A_{j}$ under the $i$-th attribute.

Based on Tables 3 and 4, using Equation (17), the intuitionistic fuzzy assessment value $\tilde{x}_{j i}$ of the alternative $A_{j}$ under the $i$-th attribute was calculated as $\tilde{x}_{11}=(0.405,0.433), \tilde{x}_{12}=(0.876,0.099), \tilde{x}_{13}$ 
Table 4

Weights of attributes

\begin{tabular}{lccccc}
\hline $\begin{array}{l}\text { Attri- } \\
\text { butes }\end{array}$ & $\begin{array}{c}\text { Overall } \\
\text { Weights }\end{array}$ & $\begin{array}{c}\text { Sub- } \\
\text { attributes }\end{array}$ & $\begin{array}{c}\text { Intuitionistic } \\
\text { Fuzzy } \\
\text { Entropy }\end{array}$ & $\begin{array}{c}\text { Entropy } \\
\text { Weights }\end{array}$ & $\begin{array}{c}\text { Relative } \\
\text { Weights }\end{array}$ \\
\hline$C_{1}$ & 0.170 & $C_{11}$ & 0.445 & 0.052 & 0.304 \\
& & $C_{12}$ & 0.319 & 0.063 & 0.373 \\
& & $C_{13}$ & 0.412 & 0.055 & 0.323 \\
$C_{2}$ & 0.171 & $C_{21}$ & 0.338 & 0.062 & 0.361 \\
& & $C_{22}$ & 0.425 & 0.054 & 0.314 \\
& & $C_{23}$ & 0.406 & 0.055 & 0.325 \\
$C_{3}$ & 0.181 & $C_{31}$ & 0.376 & 0.058 & 0.321 \\
& & $C_{32}$ & 0.453 & 0.051 & 0.282 \\
& & $C_{33}$ & 0.229 & 0.072 & 0.397 \\
$C_{4}$ & 0.121 & $C_{41}$ & 0.316 & 0.064 & 0.528 \\
& & $C_{42}$ & 0.389 & 0.057 & 0.472 \\
$C_{5}$ & 0.226 & $C_{51}$ & 0.243 & 0.071 & 0.312 \\
& & $C_{52}$ & 0.094 & 0.084 & 0.374 \\
& & $C_{53}$ & 0.239 & 0.071 & 0.314 \\
$C_{6}$ & 0.131 & $C_{61}$ & 0.363 & 0.059 & 0.453 \\
& & $C_{62}$ & 0.231 & 0.072 & 0.547 \\
\hline
\end{tabular}

$=(0.900,0.083), \quad \tilde{x}_{14}=(0.763,0.164), \quad \tilde{x}_{15}=$ $(0.872,0.102), \tilde{x}_{16}=(0.703,0.189) ; \tilde{x}_{21}=(0.832$, $0.127), \tilde{x}_{22}=(0.517,0.313), \tilde{x}_{23}=(0.517,0.294)$, $\tilde{x}_{24}=(0.950,0.050), \tilde{x}_{25}=(0.755,0.214), \tilde{x}_{26}=$ $(0.703,0.189) ; \tilde{x}_{31}=(0.756,0.166), \tilde{x}_{32}=(0.688$, $0.197), \tilde{x}_{33}=(0.111,0.859), \tilde{x}_{34}=(0.510,0.302)$, $\tilde{x}_{35}=(0.257,0.629), \tilde{x}_{36}=(0.905,0.083)$.

Step 4: Calculate the intuitionistic fuzzy assessment score $S_{L}\left(\tilde{x}_{j i}\right)$ of the alternative $A_{j}$ under the $i$-th attribute.

According to the intuitionistic fuzzy assessment value $\tilde{x}_{j i}$ of the alternative $A_{j}$ under the $i$-th attribute, with Equation (18), the intuitionistic fuzzy assessment score $S_{L}\left(\tilde{x}_{j i}\right)$ of the alternative $A_{j}$ under the $i$-th attribute was calculated as $S_{L}\left(\tilde{x}_{11}\right)=0.4709$, $S_{L}\left(\tilde{x}_{12}\right)=0.8976, \quad S_{L}\left(\tilde{x}_{13}\right)=0.9151, \quad S_{L}\left(\tilde{x}_{14}\right)=$ $0.8192, S_{L}\left(\tilde{x}_{15}\right)=0.8949, S_{L}\left(\tilde{x}_{16}\right)=0.7789 ; S_{L}$ $\left(\tilde{x}_{21}\right)=0.8662, \quad S_{L}\left(\tilde{x}_{22}\right)=0.6048, \quad S_{L}\left(\tilde{x}_{23}\right)=$ $0.6148, S_{L}\left(\tilde{x}_{24}\right)=0.9500, S_{L}\left(\tilde{x}_{25}\right)=0.7786, S_{L}$ $\left(\tilde{x}_{26}\right)=0.7789 ; \quad S_{L}\left(\tilde{x}_{31}\right)=0.8147, \quad S_{L}\left(\tilde{x}_{32}\right)=$ $0.7671, S_{L}\left(\tilde{x}_{33}\right)=0.1140, S_{L}\left(\tilde{x}_{34}\right)=0.6054, S_{L}$ $\left(\tilde{x}_{35}\right)=0.2868, S_{L}\left(\tilde{x}_{36}\right)=0.9156$.

Step 5: Determine the intuitionistic fuzzy interaction degree $S\left(\tilde{\xi}_{i j}\right)$ between $C_{i}$ and $C_{j}(i \neq j)$.

According to step 2 in Section 3, three experts employed the IFN $\alpha=\left(\mu_{\alpha}, v_{\alpha}\right)$ to assess the interactivity between $C_{i}$ and $C_{j}(i \neq j)\left(C_{6}^{2}\right.$ pairs in total $)$ respectively. Thus, the individual assessment result $\tilde{\xi}_{i j l}$ of the interactivity between $C_{i}$ and $C_{j}(i \neq j)$ was then given, as shown in Table 5.

According to step 3 in Section 3, given the weight vector $W_{D}=(0.3976,0.3012,0.3012)$ of the experts, using Equation (8) to aggregate the opinions of three experts, the group assessment result $\tilde{\xi}_{i j}$ of the interactivity between $C_{i}$ and $C_{j}(i \neq j)$ was obtained (see Table 5). Taking $\tilde{\xi}_{12}$ as an example, we had

$$
\begin{aligned}
\tilde{\xi}_{12}= & \left(1-(1-0.50)^{0.3976} \times(1-0.65)^{0.3012}\right. \\
& \times(1-0.50)^{0.3012}, 0.30^{0.3976} \times 0.20^{0.3012} \\
& \left.\times 0.30^{0.3012}\right)=(0.551,0.266) .
\end{aligned}
$$

With Equation (9), the intuitionistic fuzzy interaction degree $S\left(\tilde{\xi}_{i j}\right)$ between $C_{i}$ and $C_{j}(i \neq j)$ was calculated, as shown in Table 5. Taking $S\left(\tilde{\xi}_{12}\right)$ as an example, we had $S\left(\tilde{\xi}_{12}\right)=0.551-0.266=0.285$.

Step 6: Calculate the Möbius transformation coefficients $m_{i}$ and $m_{i j}$ of attributes.

Based on Tables 4 and 5, using Equation (10), the Möbius transformation coefficients $m_{i}$ and $m_{i j}$ of attributes were calculated, as shown in Table 6, where $P=1.125$. Taking $m_{12}$ as an example, we had

$$
m_{12}=(0.285 \times 0.170 \times 0.171) / 1.125=0.007 .
$$

Step 7: Identify the 2-order additive fuzzy measure $g_{K}$.

Based on Table 6, using Equation (11), the 2-order additive fuzzy measure $g_{K}$ was calculated, as shown in Table 7. Taking $g_{\{1,2\}}$ as an example, we had

$$
g_{\{1,2\}}=0.151+0.152+0.007=0.310 .
$$

Step 8: Calculate the Choquet fuzzy integral values and the ranking of alternatives.

Take the alternative $A_{1}$ as an example. Reordering the intuitionistic fuzzy assessment score $S_{L}\left(\tilde{x}_{1 i}\right)(i=$ $1,2, \cdots, 6)$ of alternative $A_{1}$ under the $i$-th attribute from small to large, we can get

$$
\begin{aligned}
& S_{L}\left(\tilde{x}_{11}\right)<S_{L}\left(\tilde{x}_{16}\right)<S_{L}\left(\tilde{x}_{14}\right)<S_{L}\left(\tilde{x}_{15}\right) \\
& \quad<S_{L}\left(\tilde{x}_{12}\right)<S_{L}\left(\tilde{x}_{13}\right) .
\end{aligned}
$$

It can be denoted by

$$
\begin{aligned}
& S_{L}\left(\tilde{x}_{1(1)}\right)<S_{L}\left(\tilde{x}_{1(2)}\right)<S_{L}\left(\tilde{x}_{1(3)}\right)<S_{L}\left(\tilde{x}_{1(4)}\right) \\
& \quad<S_{L}\left(\tilde{x}_{1(5)}\right)<S_{L}\left(\tilde{x}_{1(6)}\right) .
\end{aligned}
$$

Substituting the $S_{L}\left(\tilde{x}_{1(i)}\right)$ and the 2-order additive fuzzy measure $g_{K}$ into Equation (7), the Choquet fuzzy integral value of the alternative $A_{1}$ was 
Table 5

Intuitionistic fuzzy interaction degrees between attributes

\begin{tabular}{|c|c|c|c|c|c|}
\hline \multirow{2}{*}{$\begin{array}{l}\text { Attributes } C_{i} \\
\text { and } C_{j}(i \neq j)\end{array}$} & \multicolumn{3}{|c|}{ Individual Assessment Results of Interactivity } & \multirow{2}{*}{$\begin{array}{c}\text { Group Assessment } \\
\text { Results of Interactivity }\end{array}$} & \multirow{2}{*}{$\begin{array}{l}\text { Intuitionistic Fuzzy } \\
\text { Interaction Degrees }\end{array}$} \\
\hline & Expert 1 & Expert 2 & Expert 3 & & \\
\hline$\left\{C_{1}, C_{2}\right\}$ & $(0.50,0.30)$ & $(0.65,0.20)$ & $(0.50,0.30)$ & $(0.551,0.266)$ & 0.285 \\
\hline$\left\{C_{1}, C_{3}\right\}$ & $(0.65,0.20)$ & $(0.80,0.15)$ & $(0.80,0.15)$ & $(0.750,0.168)$ & 0.582 \\
\hline$\left\{C_{1}, C_{4}\right\}$ & $(0.50,0.30)$ & $(0.65,0.20)$ & $(0.50,0.30)$ & $(0.551,0.266)$ & 0.285 \\
\hline$\left\{C_{1}, C_{5}\right\}$ & $(0.50,0.30)$ & $(0.65,0.20)$ & $(0.50,0.30)$ & $(0.551,0.266)$ & 0.285 \\
\hline$\left\{C_{1}, C_{6}\right\}$ & $(0.50,0.30)$ & $(0.50,0.50)$ & $(0.50,0.30)$ & $(0.500,0.350)$ & 0.150 \\
\hline$\left\{C_{2}, C_{3}\right\}$ & $(0.65,0.20)$ & $(0.80,0.15)$ & $(0.80,0.15)$ & $(0.750,0.168)$ & 0.582 \\
\hline$\left\{C_{2}, C_{4}\right\}$ & $(0.50,0.30)$ & $(0.50,0.30)$ & $(0.65,0.20)$ & $(0.551,0.266)$ & 0.285 \\
\hline$\left\{C_{2}, C_{5}\right\}$ & $(0.50,0.30)$ & $(0.50,0.30)$ & $(0.65,0.20)$ & $(0.551,0.266)$ & 0.285 \\
\hline$\left\{C_{2}, C_{6}\right\}$ & $(0.50,0.30)$ & $(0.50,0.50)$ & $(0.50,0.30)$ & $(0.500,0.350)$ & 0.150 \\
\hline$\left\{C_{3}, C_{4}\right\}$ & $(0.65,0.20)$ & $(0.80,0.15)$ & $(0.65,0.20)$ & $(0.704,0.183)$ & 0.521 \\
\hline$\left\{C_{3}, C_{5}\right\}$ & $(0.50,0.30)$ & $(0.65,0.20)$ & $(0.65,0.20)$ & $(0.596,0.235)$ & 0.361 \\
\hline$\left\{C_{3}, C_{6}\right\}$ & $(0.50,0.30)$ & $(0.65,0.20)$ & $(0.65,0.20)$ & $(0.596,0.235)$ & 0.361 \\
\hline$\left\{C_{4}, C_{5}\right\}$ & $(0.50,0.30)$ & $(0.65,0.20)$ & $(0.65,0.20)$ & $(0.596,0.235)$ & 0.361 \\
\hline$\left\{C_{4}, C_{6}\right\}$ & $(0.20,0.65)$ & $(0.15,0.80)$ & $(0.15,0.80)$ & $(0.170,0.736)$ & -0.566 \\
\hline$\left\{C_{5}, C_{6}\right\}$ & $(0.50,0.30)$ & $(0.50,0.50)$ & $(0.50,0.30)$ & $(0.500,0.350)$ & 0.150 \\
\hline
\end{tabular}

Table 6

Calculation results of Möbius transformation coefficients

\begin{tabular}{lccccc}
\hline $\begin{array}{l}\text { Möbius Transfor- } \\
\text { mation Coefficients }\end{array}$ & Coefficient Values & $\begin{array}{c}\text { Möbius Transfor- } \\
\text { mation Coefficients }\end{array}$ & Coefficient Values & $\begin{array}{c}\text { Möbius Transfor- } \\
\text { mation Coefficients }\end{array}$ & \begin{tabular}{c} 
Coefficient Values \\
\hline$m_{1}$
\end{tabular} \\
$m_{2}$ & 0.151 & $m_{13}$ & 0.016 & $m_{26}$ & 0.003 \\
$m_{3}$ & 0.152 & $m_{14}$ & 0.005 & $m_{34}$ & 0.010 \\
$m_{4}$ & 0.161 & $m_{15}$ & 0.010 & $m_{35}$ & 0.013 \\
$m_{5}$ & 0.108 & $m_{16}$ & 0.003 & $m_{36}$ & 0.008 \\
$m_{6}$ & 0.201 & $m_{23}$ & 0.016 & $m_{45}$ & 0.009 \\
$m_{12}$ & 0.116 & $m_{24}$ & 0.005 & $m_{46}$ & -0.008 \\
\hline
\end{tabular}

Table 7

Calculation results of 2-order additive fuzzy measure

\begin{tabular}{lccccccc}
\hline$K$ & $g_{K}$ & $K$ & $g_{K}$ & $K$ & $g_{K}$ & $K$ & $g_{K}$ \\
\hline$\{\varnothing\}$ & 0.000 & $\{3,4\}$ & 0.279 & $\{2,3,4\}$ & 0.452 & $\{1,3,4,5\}$ & 0.683 \\
$\{1\}$ & 0.151 & $\{3,5\}$ & 0.375 & $\{2,3,5\}$ & 0.553 & $\{1,3,4,6\}$ & 0.570 \\
$\{2\}$ & 0.152 & $\{3,6\}$ & 0.285 & $\{2,3,6\}$ & 0.456 & $\{1,3,5,6\}$ & 0.683 \\
$\{3\}$ & 0.161 & $\{4,5\}$ & 0.317 & $\{2,4,5\}$ & 0.484 & $\{1,4,5,6\}$ & 0.599 \\
$\{4\}$ & 0.108 & $\{4,6\}$ & 0.216 & $\{2,4,6\}$ & 0.376 & $\{2,3,4,5\}$ & 0.684 \\
$\{5\}$ & 0.201 & $\{5,6\}$ & 0.321 & $\{2,5,6\}$ & 0.486 & $\{2,3,4,6\}$ & 0.571 \\
$\{6\}$ & 0.116 & $\{1,2,3\}$ & 0.503 & $\{3,4,5\}$ & 0.501 & $\{2,3,5,6\}$ & 0.684 \\
$\{1,2\}$ & 0.310 & $\{1,2,4\}$ & 0.428 & $\{3,4,6\}$ & 0.395 & $\{2,4,5,6\}$ & 0.600 \\
$\{1,3\}$ & 0.328 & $\{1,2,5\}$ & 0.531 & $\{3,5,6\}$ & 0.503 & $\{3,4,5,6\}$ & 0.621 \\
$\{1,4\}$ & 0.264 & $\{1,2,6\}$ & 0.433 & $\{4,5,6\}$ & 0.430 & $\{1,2,3,4,5\}$ & 0.874 \\
$\{1,5\}$ & 0.362 & $\{1,3,4\}$ & 0.451 & $\{1,2,3,4\}$ & 0.631 & $\{1,2,3,4,6\}$ & 0.753 \\
$\{1,6\}$ & 0.271 & $\{1,3,5\}$ & 0.552 & $\{1,2,3,5\}$ & 0.737 & $\{1,2,3,5,6\}$ & 0.871 \\
$\{2,3\}$ & 0.329 & $\{1,3,6\}$ & 0.455 & $\{1,2,3,6\}$ & 0.633 & $\{1,2,4,5,6\}$ & 0.776 \\
$\{2,4\}$ & 0.265 & $\{1,4,5\}$ & 0.483 & $\{1,2,4,5\}$ & 0.658 & $\{1,3,4,5,6\}$ & 0.806 \\
$\{2,5\}$ & 0.363 & $\{1,4,6\}$ & 0.375 & $\{1,2,4,6\}$ & 0.543 & $\{2,3,4,5,6\}$ & 0.807 \\
$\{2,6\}$ & 0.271 & $\{1,5,6\}$ & 0.485 & $\{1,2,5,6\}$ & 0.657 & $\{1,2,3,4,5,6\}$ & 1.000 \\
\hline
\end{tabular}

calculated as

$$
\begin{aligned}
& H_{1}=(0.4709-0.0000) \times 1.000+(0.7789-0.4709) \\
& \times 0.807+(0.8192-0.7789) \times 0.684+(0.8949-0.8192) \\
& \times 0.553+(0.8976-0.8949) \times 0.329+(0.9151-0.8976) \\
& \times 0.161=0.7927 .
\end{aligned}
$$

Similarly, we can also obtain $H_{2}=0.7652$ and $H_{3}=0.5157$. Since $H_{1}>H_{2}>H_{3}$, then the ranking of alternatives was $A_{1} \succ A_{2} \succ A_{3}$.

That is to say, the credit status of the alternative $A_{1}$ was relatively good, and the credit status of the alternative $A_{3}$ was relatively poor. 
Table 8

Scoring interaction degrees between attributes

\begin{tabular}{lcccc}
\hline Attributes & \multicolumn{3}{c}{ Individual Scoring } & Group Scoring \\
$C_{i}$ and $C_{j}$ & \multicolumn{3}{c}{ Interaction Degrees } & $\begin{array}{c}\text { Interaction } \\
\text { Degrees }\end{array}$ \\
\cline { 2 - 4 }$i \neq j)$ & Expert 1 & Expert 2 & Expert 3 & 0.23 \\
$\left\{C_{1}, C_{2}\right\}$ & 0.20 & 0.30 & 0.20 & 0.42 \\
$\left\{C_{1}, C_{3}\right\}$ & 0.30 & 0.50 & 0.50 & 0.23 \\
$\left\{C_{1}, C_{4}\right\}$ & 0.20 & 0.30 & 0.20 & 0.23 \\
$\left\{C_{1}, C_{5}\right\}$ & 0.20 & 0.30 & 0.20 & 0.14 \\
$\left\{C_{1}, C_{6}\right\}$ & 0.20 & 0.00 & 0.20 & 0.42 \\
$\left\{C_{2}, C_{3}\right\}$ & 0.30 & 0.50 & 0.50 & 0.23 \\
$\left\{C_{2}, C_{4}\right\}$ & 0.20 & 0.20 & 0.30 & 0.23 \\
$\left\{C_{2}, C_{5}\right\}$ & 0.20 & 0.20 & 0.30 & 0.14 \\
$\left\{C_{2}, C_{6}\right\}$ & 0.20 & 0.00 & 0.20 & 0.36 \\
$\left\{C_{3}, C_{4}\right\}$ & 0.30 & 0.50 & 0.30 & 0.26 \\
$\left\{C_{3}, C_{5}\right\}$ & 0.20 & 0.30 & 0.30 & 0.26 \\
$\left\{C_{3}, C_{6}\right\}$ & 0.20 & 0.30 & 0.30 & 0.26 \\
$\left\{C_{4}, C_{5}\right\}$ & 0.20 & 0.30 & 0.30 & -0.42 \\
$\left\{C_{4}, C_{6}\right\}$ & -0.30 & -0.50 & -0.50 & 0.14 \\
$\left\{C_{5}, C_{6}\right\}$ & 0.20 & 0.00 & 0.20 &
\end{tabular}

Note: The weighted arithmetic mean method was employed to aggregate the opinions of three experts.

\subsection{Comparative analysis}

For comparison, we also replaced the intuitionistic fuzzy interaction degrees between attributes (see Table 5) in step 5 of Section 5.2 with the scoring interaction degrees between attributes (see Table 8) determined by the interaction degrees scoring method [13]. Ceteris paribus, the Choquet fuzzy integral values of alternatives were calculated as $H_{1}^{\prime}=0.7946$, $H_{2}^{\prime}=0.7686, H_{3}^{\prime}=0.5244$. Since $H_{1}^{\prime}>H_{2}^{\prime}>H_{3}^{\prime}$, then the ranking of alternatives was $A_{1} \succ A_{2} \succ A_{3}$.

We further investigated the discrimination of the MADM method based on the intuitionistic fuzzy interaction degrees (hereinafter referred to as Method 1) for alternatives, as well as the MADM method based on the scoring interaction degrees (hereinafter referred to as Method 2). Adopting the algorithm of discrimination given in [33], the discrimination of Method 1 for alternatives was calculated as

$$
\begin{aligned}
& \rho=\frac{0.7927-0.7652}{0.7927}+\frac{0.7927-0.5157}{0.7927} \\
& +\frac{0.7652-0.5157}{0.7652}=0.7102 .
\end{aligned}
$$

Simultaneously, the discrimination of Method 2 for alternatives was calculated as

$$
\begin{aligned}
& \rho^{\prime}=\frac{0.7946-0.7686}{0.7946}+\frac{0.7946-0.5244}{0.7946} \\
& +\frac{0.7686-0.5244}{0.7686}=0.6903 .
\end{aligned}
$$

Although the ranking result of Method 2 is consistent with that of Method 1, the discrimination of Method 1 for alternatives is higher than that of Method 2. Which means that the decision-making result of Method 1 is better than that of Method 2.

\section{Discussion}

From the results and analysis of the previous section, we observed that Method 1 was able to obtain the higher discrimination value than Method 2 (Method 1 was 0.7102 , and Method 2 was 0.6903), and the slightly lower Choquet fuzzy integral mean value than Method 2 (Method 1 was 0.6912, and Method 2 was 0.6959).

Compared with Method 2, Method 1 can obtain the higher discrimination value. Since the variance of interaction degrees determined by Method 1 (its value was equal to 0.0684 ) was higher than that of Method 2 (its value was equal to 0.0355). According to Equation (6), the variances of $m_{i}$ and $m_{i j}$ increase with the increase of the variance of interaction degrees. From Equations (5) and (7), we can further see that the variance of $g(K)$ and the variance of $H$ also increase correspondingly. Thus, according to the algorithm of discrimination [33], the discrimination value becomes larger.

Compared with Method 2, Method 1 can obtain the slightly lower Choquet fuzzy integral mean value. Since the average value of interaction degrees determined by Method 1 (its value was equal to 0.2718 ) was higher than that of Method 2 (its value was equal to 0.2087 ). According to Equation (6), when the average value of interaction degrees increases, the mean value of $P$ increases, meanwhile, the mean value of $m_{i}$ decreases and the mean value of $m_{i j}$ increases. However, because $\left|m_{i}\right| \gg\left|m_{i j}\right|$, from Equation (5), we can further see that the mean value of $g(K)$ also decreases correspondingly. Thus, according to Equation (7), the mean value of $H$ becomes smaller.

The variance or average value of interaction degrees determined by Method 1 was higher than that of Method 2, which was closely related to that the experts used the IFN to assess the interactivity between attributes, so it can fully express their opinions.

\section{Conclusion}

In this paper, the proposed method defines the interactivity between attributes by using the 
supermodular game theory, so that the interaction between attributes is easier to explain and understand, which lays a solid foundation for experts to qualitatively assess the interactivity between attributes. The proposed method allows the experts to assess the interactivity between attributes by using the IFN, which better preserves the assessment information of experts and embodies the fuzziness and hesitation of interactivity assessment. Furthermore, the proposed method uses the IFWA to aggregate the opinions of all experts, which considers the weights of experts, thereby ensuring the rationality of decision-making. In addition, the proposed method uses the score function of IFN to define and calculate the intuitionistic fuzzy interaction degree between attributes, so the transformation from qualitative description to quantitative characterization is finally realized. Therefore, using the IFSs, this work successfully solves the problem that it is difficult to quantitatively assess the interactivity between attributes in the identification process of 2-order additive fuzzy measure.

This work proposed a 2-order additive fuzzy measure identification method based on IFSs. Obviously, on the one hand, compared with the objective method describing and dealing with the interactivity between attributes (as in [7, 16] and [17]), the proposed method has subjectivity. On the other hand, compared with the subjective method describing and dealing with the interactivity between attributes (as in [9-14] and [15]), the proposed method has fuzziness and hesitation.

Future application example analysis will consider increasing the number of samples and experts to improve the persuasiveness of application example analysis results and the stability of interactivity assessment. Furthermore, because the sample time may affect the analysis results of application example, it is necessary to update the sample data from 2016 to 2020 in the future. In addition, Method 1 should be compared with other methods except Method 2, such as the diamond pairwise comparisons method $[9,10$, 14], proportional scaling method [11], multicriteria correlation preference information method [12], etc.

According to the supermodular game theory, Definition 9 gives the definition of interactivity between attributes. However, this definition is still relatively general. The detailed theoretical analysis of the connotation of interactivity between attributes needs to be completed in the future. The existing 2-order additive fuzzy measure identification methods use the subjective methods and the objective methods to describe and deal with the interactivity between attributes. Thus, combining the results of subjective methods and objective methods is one of the main research directions in the future. In addition, constructing a Group Decision Support System will reduce the complexity of Method 1 in practical application.

\section{Acknowledgments}

To the Regional Project of National Natural Science Foundation of China (71861003) and the Innovative Exploration and New Academic Seedlings Project of Guizhou University of Finance and Economics (Guizhou-Science Cooperation Platform Talents [2018] 5774-016) for their support.

\section{References}

[1] J.L. Marichal, An axiomatic approach of the discrete Choquet integral as a tool to aggregate interacting criteria, IEEE Transactions on Fuzzy Systems 8(6) (2000), 800-807.

[2] M. Sugeno, Theory of Fuzzy Integrals and Its Application, Ph.D. Dissertation, Tokyo Institute of Technology (1974).

[3] T. Murofushi and M. Sugeno, A theory of fuzzy measures: Representations, the Choquet integral, and null sets, Journal of Mathematical Analysis and Applications 159(2) (1991), 532-549.

[4] Z.Y. Wang and G.J. Klir, Fuzzy Measure Theory, Plenum Publishing Corporation, New York, (1992).

[5] K. Ishii and M. Sugeno, A Model of Human Evaluation Process Using Fuzzy Measure, International Journal of Man-Machine Studies 22(1) (1985), 19-38.

[6] M. Grabisch, K-order Additive Discrete Fuzzy Measures and Their Representation, Fuzzy Sets and Systems 92(2) (1997), 167-189.

[7] J.Z. Wu and Q. Zhang, 2-order additive fuzzy measures identification method based on maximum entropy principle, Systems Engineering and Electronics 32(11) (2010), 2346-2351.

[8] B. Mayag and D. Bouyssou, Necessary and possible interaction between criteria in a 2-additive Choquet integral model, European Journal of Operational Research 283(1) (2020), 308-320.

[9] E. Takahagi, A fuzzy Measure Identification Method by Diamond Pairwise Comparisons and $\varnothing(\mathrm{s})$ Transformation, Fuzzy Optimization and Decision Making 7(3) (2008), 219-232.

[10] J.Z. Wu and Q. Zhang, Multicriteria decision making method based on 2-order additive fuzzy measures, Systems Engineering-Theory \& Practice 30(7) (2010), 1229-1237.

[11] Z. Zhang, K. Fang and M. Yang, Method for simulation credibility evaluation based on 2-additive fuzzy measures, Control and Decision 28(1) (2013), 147-151.

[12] J.Z. Wu, S.L. Yang, Q. Zhang and S. Ding, 2-additive Capacity Identification Methods from Multicriteria Correlation Preference Information, IEEE Transactions on Fuzzy Systems 23(6) (2015), 2094-2106. 
[13] Z.P. Chang and L.S. Cheng, Grey Fuzzy Integral Correlation Degree Decision Model, Chinese Journal of Management Science 23(11) (2015), 105-111.

[14] S.W. Lin and M.A. Jerusalem, Hybrid method for assessing marketable fashion designs: diamond pairwise comparison combined with the Choquet integral, Textile Research Journal 88(13) (2018), 1531-1542.

[15] C. Kadaifci, U. Asan and E. Bozdag, A new 2-additive Choquet integral based approach to qualitative cross-impact analysis considering interaction effects, Technological Forecasting and Social Change 158 (2020), 120131.

[16] Z.P. Chang, L.S. Cheng and J.S. Liu, Multi-Attribute Decision Making Method Based on Mahalanobis-Taguchi System and 2-additive Choquet integral, Journal of Industrial Engineering and Engineering Management 30(1) (2016), 133-139.

[17] Z.P. Chang, L.S. Cheng, S.Z. Lu and X.Z. Wang, Exit decision model of public rental housing based on 2-order additive fuzzy measures, Journal of Nanjing University of Science and Technology (Nature Science) 41(1) (2017), 132-138.

[18] K.T. Atanassov, Intuitionistic Fuzzy Sets, Fuzzy Sets and Systems 20(1) (1986), 87-96.

[19] Z.S. Xu, Intuitionistic fuzzy information aggregation theory and its application, Science Press, Beijing, (2008).

[20] C.Q. Tan, TOPSIS Multiple Attribute Decision Making Based on Interval-valued Intuitionistic Fuzzy Sets, Fuzzy Systems and Mathematics 24(1) (2010), 92-97.

[21] K.R. Wang and H. Yang, Multiple Attribute Decision Making Method Based on Intuitionistic Fuzzy Sets, Fuzzy Systems and Mathematics 24(3) (2010), 114-118.

[22] R. Verma and J.M. Merigó, Multiple attribute group decision making based on 2-dimension linguistic intuitionistic fuzzy aggregation operators, Soft Computing 24 (2020), $17377-17400$.
[23] T. Murofushi and M. Sugeno, An Interpretation of Fuzzy Measures and the Choquet Integral as an Integral with Respect to a Fuzzy Measure, Fuzzy Sets and Systems 29(2) (1989), 201-227.

[24] Z.S. Xu, Intuitionistic Fuzzy Aggregation Operators, IEEE Transactions on Fuzzy Systems 15(6) (2007), 1179-1187.

[25] Y. Wang and Y.J. Lei, A technique for constructing intuitionistic fuzzy entropy, Control and Decision 22(12) (2007), 1390-1394.

[26] S.M. Chen and J.M. Tan, Handling Multi-criteria Fuzzy Decision-making Problems Based on Vague Set Theory, Fuzzy Sets and Systems 67(2) (1994), 163-172.

[27] H.W. Liu, Vague Set Methods of Multicriteria Fuzzy Decision Making, Systems Engineering-Theory \& Practice 24(5) (2004), 103-109.

[28] D.M. Topkis, Minimizing a submodular function on a lattice, Operations Research 26(2) (1978), 305-321.

[29] W.H. Qiu, Manage decision and entropy, Mechanical Industry Press, Beijing, (2002).

[30] J.N. Meng, N. Fang, B.S. Liu and B. Xue, Sustainability Evaluation Model for Urban Infrastructure Projects Based on Intuitionistic Fuzzy Sets, Fuzzy Systems and Mathematics 29(1) (2015), 167-174.

[31] M. Zhang and X.P. Wang, Evaluation on Risk of Intellectual Property Pledge Financing for Big Data Enterprises, Science and Technology Management Research 39(21) (2019), $61-66$.

[32] Y.H. Li, Evaluation and selection of strategic emerging industries based on Fuzzy AHP considering the weight of experts: An empirical analysis of Tangshan, China Collective Economy (6) (2017), 60-62.

[33] H.F. Ke, Y.G. Chen and B. Xia, An Algorithm of Multiple Criteria Decision-Making Based on Similarity to Ideal Grey Relational Projection, Acta Electronica Sinica 35(9) (2007), $1757-1761$ 


\section{Appendix A}

In step 1 of Section 5.2, the weight vector of experts was calculated by using the cycle mutual evaluation method [32]. Its calculation principle is as follows:

Suppose there are $p$ experts, $b_{i j}$ is the mutual evaluation weight given by expert $i$ to expert $j$, then the mutual evaluation weight matrix is $B=$ $\left(b_{i j}\right)_{p \times p}$, where $0 \leq b_{i j} \leq 1$, and $\sum_{j=1}^{p} b_{i j}=1$. Then the weight of expert $j$ given by the group is $q_{j}=$ $p^{-1} \sum_{i=1}^{p} b_{i j}$. Therefore, the final weights of experts are determined through the cycle evaluation. Let $t$ be the number of mutual evaluations, when $t=1$, the weight vector of experts is $q^{1}=\left(q_{1}, q_{2}, \cdots, q_{p}\right)$; when $t>1$, the weight vector of experts is $q^{t}=q^{t-1} B$, until $q^{t}$ converges to the stable value.
In this paper, the mutual evaluation weight matrix was given by three experts, it was

$$
B=\left[\begin{array}{lll}
0.50 & 0.25 & 0.25 \\
0.38 & 0.31 & 0.31 \\
0.28 & 0.36 & 0.36
\end{array}\right]
$$

When $t=1$, the weight vector of experts was $q^{1}=(0.3866,0.3067,0.3067)$; when $t=5$, the weight vector of experts converged to the stable value $q^{5}=(0.3976,0.3012,0.3012)$. Therefore, the final weight vector of experts was $W_{D}=$ $(0.3976,0.3012,0.3012)$. 